\title{
Um modelo conceptual para a cocriação de valor do turista: Uma abordagem exploratória
}

\section{A conceptual model for the co-creation of tourist value: An exploratory approach}

\author{
Pedro Miguel Carvalho \\ Instituto Politécnico de Viana do Castelo, CITUR, Portugal \\ pc@estg.ipvc.pt
}

\begin{abstract}
Resumo
A comunidade científica tem apresentado vários contributos conceptuais e estudos empíricos transdisciplinares associados à perspetiva do Service-Dominant Logic (S-D Logic), onde a indústria do turismo não é excepção. Neste sentido, é de realçar a existência de diversos trabalhos empíricos já desenvolvidos neste domínio, com especial enfoque na cocriação de valor do turista. Este artigo, enfatizando a compreensão do conceito de cocriação de valor do cliente, visa propor um modelo conceptual agregador que permita identificar as diferentes dimensões da cocriação de valor do turista, resultante dos múltiplos contributos empíricos da comunidade científica. Com efeito, este artigo para além de contribuir para uma compreensão mais abrangente das várias dimensões associadas à cocriação de valor do turista na perspetiva do S-D Logic, poderá auxiliar as organizações turísticas, a partir do modelo conceptual proposto, na avaliação do processo de cocriação de valor dos seus clientes, assim como na comunicação e concepção do design de serviço.
\end{abstract}

Palavras-chave: Cocriação de valor; service dominant-logic; cocriação do cliente.

\begin{abstract}
The scientific community has provided numerous conceptual contributions and transdisciplinary empirical studies associated with the Service-Dominant Logic (S-D Logic) perspective, where the tourism industry is no exception. In this sense, it is worth highlighting the existence of several empirical works already developed in this field, with special focus on tourist value co-creation. This article, emphasizing understanding of the concepts of customer value co-creation, aims to propose an aggregating conceptual model to identify the different dimensions of tourist value cocreation, resulting from the multiple empirical contributions of scientific community on this subject. Indeed, this article, in addition to contributing to a more comprehensive understanding of the various dimensions of the tourist value co-creation from the perspective of S-D Logic, may assist tourism organizations, based on the proposed conceptual model, in assessing the value cocreation process of its customers, as well as in the communication and conception of service design.
\end{abstract}

Keywords: Value co-creation; service dominant-logic; customer co-creation.

Dos Algarves: A Multidisciplinary e-Journal, 38-2020.

ISBN 2182-5580 @ ESGHT - University of the Algarve, Portugal.

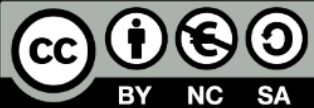

To cite this article: Carvalho, P. M. (2020). Um modelo conceptual para a cocriação de valor do turista: Uma abordagem exploratória. Dos Algarves: A Multidisciplinary e-Journal, 38, 89-104. DOI: 10.18089/DAMeJ.2020.38.4 


\section{Introdução}

Nos anos 70, Lynn Shostack (1977), num artigo publicado no Journal of Marketing apresentou, pela primeira vez, o Marketing de Serviços como um campo relevante de pesquisa. Amparados pelas correntes de pensamento do Marketing desse período, entre 1977 e 2004, uma enorme quantidade de estudos ligados a esta nova área de investigação foram publicados (cf. Berry \& Parasuraman, 1993; Grönroos \& Gummesson, 1985; Eiglier \& Langeard, 1987), discutindo conceitos e modelos baseados no serviço, com um enfoque de pesquisa orientado para a facilitação e gestão de interacções entre a empresa e o cliente (Grönroos, 2006). Todavia, em 2004, os autores norte-americanos, Vargo e Lusch (2004), também num artigo difundido no Journal of Marketing, apresentaram uma nova perspetiva para o Marketing, alicerçando os seus argumentos num conjunto de ideias-base. Para estes autores, a teoria económica tinha estado fundamentalmente ligada a unidades produzidas (produtos), que são impregnadas de valor durante o processo de produção, idealmente estandardizadas e realizadas no isolamento do cliente, com o objetivo de serem vendidas no mercado para a captação e estimulação da procura.

Segundo Vargo e Lusch (2004), estas seriam as ideias-base que tinham orientado o desenvolvimento de toda a teoria económica e de todas as disciplinas provenientes, desde o trabalho desenvolvido por Adam Smith ([1776] 2007). Contudo, estes autores refutaram a clássica orientação - output-centric -, para uma nova orientação - process-centric. Segundo esta nova lógica, o propósito da troca seria fornecer serviços, no sentido de obter reciprocamente serviços. Isto significa que o cliente e o fornecedor trocam competências especializadas, isto é, serviço por serviço. Deste modo, para estes investigadores, o serviço é sempre a fonte essencial de criação de valor, devendo os produtos ser encarados como "appliances" para o fornecimento do serviço.

Vargo, Lusch, Akaka e He (2010) argumentaram ainda que esta nova lógica, designada Service-Dominant Logic (S-D Logic), seria convergente com as teorias do marketing relacional e de serviços (ex. Gummesson, 2002), teoria do "resource-advantage" (ex. Hunt, 2000), teoria das competências core (ex. Prahalad \& Hamel, 1990) e teoria de redes (ex. Hakansson \& Snehota, 1995). Mais é de salientar que, quando a discussão do S-D Logic surgiu, Vargo e Lusch (2004) assumiram oito premissas fundacionais que foram revistas, produto do diálogo e discussão com vários académicos. A revisão mais completa das premissas expôs onze premissas fundacionais (Vargo \& Lusch, 2016).

Uma questão crucial trazida por Vargo e Lusch (2004) está associada ao significado do valor. De acordo com esta nova perspetiva, o valor é determinado pelo cliente com base no uso e não com base na utilidade. $O$ argumento apresentado pelos autores é que o valor percebido pelo cliente (no produto ou serviço) está dependente do modo como faz o uso do produto através do seu próprio conhecimento. Ora esta premissa permite justificar que o cliente é um participante ativo no processo de criação de valor, podendo mesmo ser encarado, segundo Vargo e Lusch (2006), como um cocriador de valor.

Desde então, vários contributos conceptuais e estudos empíricos têm sido apresentados pela comunidade científica associados à perspetiva do S-D Logic, sendo de realçar vários trabalhos ligados ao desenvolvimento destes conceitos, suas premissas e axiomas (ex. Vargo \& Lusch, 2006; Vargo \& Lusch, 2013; Vargo et al., 2017), novas conceptualizações da marca (ex. Brodie, Glynn \& Little, 2006; Merz, He \& Vargo, 2009) e de mercado (ex. Mele, Pels \& 
Storbacka, 2015), novas abordagens a modelos de negócio (Wieland, Hartmann \& Vargo, 2017) e à inovação em serviços (ex. Skålén, Gummerus, Koskull \& Magnusson, 2015), assim como vários estudos ligados ao desenvolvimento da cocriação de valor numa perspetiva diádica, triádica e em rede (ex. Gummesson \& Mele, 2010; Chandler \& Vargo, 2011; Grönroos \& Gummerus, 2014).

A abordagem do S-D Logic e os seus contributos conceptuais têm também merecido particular atenção no setor do turismo (ex. Park \& Vargo, 2012; Horbel, 2013) e, no âmbito desta indústria, é de realçar a existência de diversos estudos empíricos já desenvolvidos, com especial ênfase na cocriação de valor do cliente (ex. Prebensen \& Dahl, 2013; Grissemann \& Stokburger-Sauer, 2012; Buonincontri, Morvillo, Okumus, \& van Niekerk, 2017; Xu, Liu \& Lyu, 2018). Todavia, importa realçar que os estudos sobre a cocriação de valor do cliente em organizações turísticas têm também sido evidenciados pela comunidade científica, com recurso a outras abordagens teóricas, designadamente, teoria da troca social (ex. Hsiao, Lee \& Chen, 2015), customer-dominant logic (ex. Rihova, Buhalis, Moital \& Gouthro, 2015; Rihova, Buhalis, Gouthro \& Moital, 2018; Malone, McKechnie \& Tynan, 2018), cocriação da experiência (ex. Chen, 2018; Beliatskaya, 2017); social media (ex. Ge \& Gretzel, 2018; He \& Wang, 2016) e teoria da conservação dos recursos (ex. Tuan, Rajendran, Rowley \& Khai, 2019).

Embora sejam vários os autores que têm manifestado a importância de se continuarem a desenvolver mais estudos no âmbito da cocriação de valor do cliente nesta indústria (cf. Grissemann \& Stokburger-Sauer, 2012; Cha, Yi \& Bagozzi, 2016), é ainda de realçar a exiguidade de trabalhos que sistematizam o conhecimento empírico produzido pela comunidade científica (ex. Shamim \& Ghazali, 2014; Campos, Mendes, Oom do Valle \& Scott, 2015). Dada a dificuldade de compreender, numa perspetiva holística, os diferentes meandros do conhecimento sobre a cocriação de valor nesta indústria, pela magnitude de estudos realizados, urge a necessidade de sistematização para uma compreensão global desta matéria. Com efeito, justifica-se a criação de um estudo conceptual, agregador de várias abordagens empíricas, que permita a facilitação da transmissão do conhecimento empírico e das suas implicações práticas junto dos profissionais desta indústria.

Tendo por base uma rigorosa pesquisa de artigos empiricamente testados em organizações de diferentes setores na indústria do turismo, com recurso a metodologias quantitativas, este artigo tem como objetivo apresentar um modelo conceptual integrador das diferentes dimensões da cocriação de valor do cliente na perspetiva do Service-Dominant Logic.

Este estudo também apresenta implicações teóricas relevantes para a comunidade científica, contribuindo para uma compreensão mais abrangente das várias dimensões da cocriação de valor do turista (na perspetiva do S-D Logic). Por outro lado, o modelo conceptual proposto poderá auxiliar as organizações turísticas na avaliação do processo de cocriação de valor dos seus clientes e na concepção do seu design de serviço.

Assim, este artigo começa por abordar a cocriação de valor com base nesta abordagem do marketing - S-D Logic, tendo em vista apresentar um modelo conceptual agregador sobre a cocriação de valor do turista. A partir da discussão do modelo proposto, são apresentadas implicações para os profissionais desta indústria e comunidade científica, assim como orientações para futuros trabalhos de investigação. 


\section{A cocriação de valor na perspetiva do Service-Dominant Logic}

A perspetiva do S-D Logic assenta no princípio-base de que todas as economias são economias de serviços, uma vez que o serviço é a base fundamental de troca. A manufatura é apenas um caso especial de fornecimento de serviço (Vargo \& Lusch, 2004). Por isso, a visão de Vargo e Lusch (2004) altera a perspetiva de compreensão dos mercados e do marketing. Segundo Vargo et al. (2010), o propósito da troca consiste em fornecer um serviço e obter reciprocamente um serviço: trocar um serviço por outro serviço. Vargo e Lusch (2008) realçam que o serviço consiste na aplicação de recursos operant (técnicas e conhecimentos) para benefício de outra parte. Neste sentido, Vargo e Lusch $(2004,2006)$ diferenciam o conceito de recursos operant de recursos operand. Deste modo, realçam os primeiros como sendo intangíveis, dinâmicos e capazes de criar valor, referindo-se concretamente aos conhecimentos e técnicas aplicadas pelos funcionários da empresa e seus clientes. Por sua vez, os segundos deveriam ser conceptualizados, como sendo estáticos, geralmente tangíveis e que requerem ação para torná-los valiosos. Com efeito, os bens deverão ser encarados como recursos operand e transmissores de conhecimento e técnica, que são usados por outros recursos operant (ex. clientes). Por outro lado, o valor deveria ser determinado pelo cliente com base no "valor de uso" e não pelo "valor-utilidade". Ora, os produtos deverão ser encarados como aplicações dos serviços através dos quais os clientes derivam o seu "valor de uso" (Vargo \& Lusch, 2004).

A propósito desta matéria, a escola nórdica de serviços (Service Logic) realça também algumas especificidades. Desde logo, enfatiza que os fornecedores criam os recursos e os meios necessários que permitem ser possível aos clientes criar valor para eles próprios: "valor de uso" (Grönroos, 2006). Isto significa que os clientes não recebem o valor incorporado nos produtos, mas percepcionam um valor que está dependente do modo como fazem o uso dos produtos (Grönroos, 2009; Grönroos \& Gummerus, 2014). Assim, a noção de "valor de uso" inclui não só o uso do próprio produto, mas também elementos como, bens, informação, encontros pessoais, interações com sistemas e infra-estruturas, e possíveis interacções com outros clientes que, em conjunto, têm impacto na criação de valor para o cliente (Grönroos, 2006). Por outro lado, a escola nórdica de serviços não concorda com a premissa do S-D Logic "that the user and service provider are always co-creators of value, nor with the notion that the firm as service provider can influence customers' value creation by allowing customers to join the firm's processes as value co-creators" (Grönroos \& Gummerus, 2014: 212), embora reconheça que a empresa tem a oportunidade de cocriar valor com os seus clientes e que estes são os últimos criadores de valor (para eles próprios). Por isso, a empresa deverá ser encarada como uma "facilitadora" de valor (Grönroos, 2009).

Em sintonia com a perspetiva do S-D Logic, Vargo et al. (2010) acrescentam, referindo que a criação de valor envolve competências do cliente na coprodução de valor, no seu próprio contexto e a participação do cliente na coprodução da proposta de valor da empresa. Aliás, de acordo com Vargo e Akaka (2009), não pode existir valor sem o cliente incorporar as ofertas da empresa na sua vida. Por outro lado, a participação do cliente na oferta da empresa (ex. design, montagem, self-service) deverá ser conceptualizada como coprodução. Assim, o papel do cliente na "coprodução" é opcional, enquanto a "cocriação" de valor não é. Ora, segundo estes autores (Vargo \& Akaka, 2009), o valor é sempre cocriado. 
Vargo et al. (2010) e Skålén et al. (2015) sugerem que o valor cocriado é também resultado da integração de outros recursos contextualmente específicos. A título de exemplo, os benefícios de uso de um carro, não estão somente associados ao seu uso, mas também são determinados pela integração de outros recursos, tais como, estradas, combustível, habilidade de condução e pelo uso do contexto (por exemplo, a integração de atividades familiares, tais como, jogos de futebol ao fim-de-semana determina um valor de uso diferente ligado a atividades diárias relacionadas com o trabalho). Com efeito, o cliente integra a proposta de valor do prestador de serviço com outros recursos do seu próprio contexto (Park \& Vargo, 2012; Horbel, 2013).

\section{A cocriação de valor do turista: Proposta de um modelo conceptual}

A abordagem do S-D Logic no marketing turístico não está restrita à compreensão das necessidades e desejos dos clientes, implicando, por isso, o envolvimento, a colaboração e a aprendizagem através do turista. Deste modo, a organização turística terá o papel de ser uma das numerosas fontes de recursos que o turista recorre para atingir o valor desejado nas experiências, assumindo, um papel direto (executar) e indireto ou de capacitação (facilitando o self-service) (Park \& Vargo, 2012).

Por outro lado, Park e Vargo (2012) explicam que, "Tourists should be viewed no longer as recipients of value or targets but as value co-creators who participate in, influence and collaborate with the firm in relationship, utilizing their own resources" (242). Deste modo, a criação de valor é interativa, através de redes dinâmicas que envolvem vários atores (ex. empregados, turistas, outros hóspedes, tecnologias). Mais ainda, o valor é criado com os turistas e por eles definido (experienciadores), no contexto das suas próprias circunstâncias únicas (ex. valores pessoais, estilo de vida e culturas). Isto significa que não terão a mesma experiência, mesmo quando encontram uma oferta similar (Park \& Vargo, 2012).

No processo de cocriação de valor, os turistas desenvolvem relacionamentos com vários atores (empregados de mesa, residentes, atrações locais, funcionários do alojamento, transportes, etc.) e combinam uma diversidade de recursos (interagindo), para cocriar a sua experiência. Por isso, as empresas devem procurar atender às propostas de valor de todos os participantes do ecossistema (Park \& Vargo, 2012; Horbel, 2013). Por sua vez, os turistas também "fornecem" valor para a empresa aumentando a sua reputação e apoiando a conquista de novos clientes, através da concessão de "passa-palavra", assumindo parte do desenvolvimento de novos produtos e serviços, por exemplo, concedendo sugestões ou até mesmo criando significados para a marca em comunidades de clientes (Vargo, Lush, Horbel \& Wieland, 2011; Woratschek \& Horbel, 2005).

Ora, reconhecendo-se a importância que a cocriação de valor tem para as organizações turísticas e atendendo-se à multiplicidade de estudos que seguem uma abordagem puramente conceptual (ex. Shaw, Bailey \& Williams, 2011), qualitativa (ex. Malone et al., 2018; Assiouras, Skourtis, Giannopoulos, Buhalis \& Koniordos, 2019) quantitativa (ex. Prebensen, Woo \& Uysal, 2013; Xu et al., 2018) e a exiguidade de trabalhos que sistematizam o conhecimento empírico produzido (ex. Shamim \& Ghazali, 2014; Campos et al., 2015), urge a necessidade de apresentar um modelo (conceptual) agregador dos diferentes contributos que permita melhor compreender a essência da cocriação de valor e das suas diferentes dimensões. Tendo por base uma rigorosa revisão de literatura sobre a cocriação de valor numa perspetiva do S-D Logic, centrada na indústria do turismo e com recurso a 
metodologias quantitativas, é possível verificar a existência de grandes disparidades nas dimensões identificadas pelos autores (Tabela 1). 
Tabela 1. Estudos sobre a cocriação de valor do turista na perspetiva do Service-Dominant Logic

\begin{tabular}{|c|c|c|}
\hline Estudos & Dimensões da cocriação de valor & Contexto do estudo \\
\hline Prebensen, Kim \& Uysal (2016) & A cocriação foi mensurada por dois conceitos: interesse (situacional mental) e participação (física). & Empresa de turismo de inverno \\
\hline Prebensen \& Xie (2017) & $\begin{array}{l}\text { Neste estudo, a cocriação de valor é integrada no construto participação. Assim, a participação } \\
\text { inclui: (i) cocriação física; (ii) cocriação psicológica e (iii) autoperceção de mestria pessoal. }\end{array}$ & Empresa de turismo de inverno \\
\hline Sarmah, Kamboj, \& Rahman (2017) & $\begin{array}{l}\text { O conceito de cocriação estudado é duplo: envolvimento do cliente e vontade do cliente de } \\
\text { cocriar. }\end{array}$ & Hotelaria \\
\hline Busser \& Shulga (2018) & $\begin{array}{l}\text { Os autores apresentam cinco dimensões: significância, colaboração, contribuição, } \\
\text { reconhecimento e resposta afetiva. }\end{array}$ & Hotelaria e restauração \\
\hline Shulga, Busser \& Kim (2018) & $\begin{array}{l}\text { Este estudo apresenta quatro tipos de cocriação de valor: co-inovação, co-recuperação, cocriação } \\
\text { de experiência e co-marketing. }\end{array}$ & Hotelaria \\
\hline Sthapit, Coudounaris \& Björk (2018) & A cocriação compreende dimensões como a participação física e psicológica. & Lojas de venda de lembranças \\
\hline Ahn, Lee, Back \& Schmitt (2019) & $\begin{array}{l}\text { A cocriação é apresentada como um conceito multidimensional, estruturado em dois grupos: (i) } \\
\text { atitude de cocriação e (ii) comportamento de cocriação. Assim, a atitude de cocriação depende de } \\
\text { três dimensões: atitude de interação, compartilhamento de conhecimento e atitude responsiva. } \\
\text { Por sua vez, o comportamento de cocriação depende do comportamento da participação e do } \\
\text { comportamento da cidadania. }\end{array}$ & Hotelaria \\
\hline Huang \& Choi (2019) & $\begin{array}{l}\text { Para medir a qualidade da experiência no processo de cocriação de valor, foram apresentadas } \\
\text { quatro dimensões: (i) interação social, (ii) interação com funcionários, (iii) relacionamento com } \\
\text { acompanhantes e (iv) envolvimento do turista com a atividade. }\end{array}$ & Turismo de cruzeiros \\
\hline Assiouras et al. (2019) & $\begin{array}{l}\text { A cocriação de valor depende de duas dimensões: valor em uso e coprodução. A coprodução é } \\
\text { analisada em três subdimensões: compartilhamento de conhecimento, equidade e interação. }\end{array}$ & Painel online de turistas \\
\hline Rather, Hollebeek \& Islam (2019) & $\begin{array}{l}\text { Neste estudo, a cocriação de valor compreende duas dimensões: (i) Valor em uso e (ii) } \\
\text { coprodução. }\end{array}$ & Destino turístico famoso na Índia \\
\hline
\end{tabular}


Assim, no sector da hotelaria, Sarmah, Kamboj e Rahman (2017) explicam que a cocriação de valor inclui o envolvimento do cliente e a vontade do cliente de cocriar. Para esses autores, o envolvimento do cliente é referido como "the degree to which customers take part in creating, producing and delivering new services" (2017: 2652), bem como os clientes dispõem-se a cocriar "if they consider their involvement in co-creation activities as benefits rather than costs" (2017: 2653). Por sua vez, Busser e Shulga (2018), destacam cinco dimensões associadas à cocriação de valor: reconhecimento (a perceção do turista que está a obter reconhecimento intrínseco ou extrínseco por estar implicado com práticas de cocriação de valor), significância (a crença individual do turista da importância do seu envolvimento na cocriação de valor); resposta afetiva (toda a reação emocional do turista pela cocriação, tal como, interesse, alegria, felicidade e diversão); colaboração (isto é, cooperação para ganho mútuo entre dois ou mais atores envolvidos na cocriação de valor) e contribuição (partilha de recursos tangíveis e intangíveis). No mesmo contexto de estudo, Shulga et al.(2018) realçam quatro tipos de cocriação de valor, designadamente co-inovação (envolve o cliente e o provedor de serviços no desenvolvimento de produtos ou serviços); correcuperação (processo de colaboração conjunta entre o cliente e o provedor de serviços para criar uma solução para a falha de serviço); cocriação de experiência (processo pelo qual os clientes cooperam com os provedores de serviços para definir, moldar e personalizar as suas experiências por meio de configurações e opções) e comarketing (especialmente em termos da conexão do valor em uso ao valor da marca).

Ainda neste setor, Ahn et al. (2019) apresentam a cocriação de valor como um conceito multidimensional, estruturado em dois grupos, nomeadamente (i) atitude de cocriação e (ii) comportamento de cocriação. Para estes investigadores, a atitude de cocriação depende de três dimensões: atitude de interação, compartilhamento de conhecimento e atitude responsiva. Baseando-se no trabalho de Yi e Gong (2013), Ahn et al. (2019) referem que o comportamento de cocriação de valor depende do comportamento da participação do turista e do comportamento de cidadania. A propósito, Yi e Gong (2013) explicam que o comportamento da participação é determinado por fatores como a procura de informação (os turistas recolhem dados para clarificar os requisitos do serviço e reduzir a incerteza ambiental), contribuição com recursos (partilham os seus recursos tangíveis e intangíveis para atingir os resultados desejados), comportamento responsável (os turistas revelam que estão conscientes das suas próprias responsabilidades e têm vontade de cooperar, cumprindo as regras e aceitando as orientações dos funcionários) e interação pessoal (estabelecem interações pessoais com os funcionários). Estes autores salientam ainda que o comportamento de cidadania, é influenciado pelo feedback do turista (fornecem ativamente sugestões que serão benéficas para a melhoria do serviço), a advocacia (os clientes promovem ativamente a organização e os seus funcionários junto de pessoas fora da organização, contribuindo para a melhoria da reputação e avaliação de produtos e serviços da organização), a ajuda (os turistas tomam a iniciativa de auxiliar outros clientes) e a tolerância (manifestam maior tolerância em situações em que o serviço prestado pelos funcionários não corresponde às expectativas).

Outros trabalhos foram desenvolvidos, noutros contextos de estudo. Por exemplo, em estudos desenvolvidos em empresas de turismo de inverno, Prebensen et al. (2016) avaliam 
a cocriação de valor a partir dos conceitos: interesse (situacional mental) e participação (física), assim como Prebensen e Xie (2017) conceptualizam a cocriação de valor integrandoa no construto participação. Para estes autores, a participação inclui: a cocriação física, a cocriação psicológica e a autoperceção de mestria pessoal (isto é, como os consumidores percecionam os seus conhecimentos e habilidades em relação ao desempenho de uma determinada atividade).

Huang e Choi (2019), num estudo aplicado ao turismo de cruzeiros, apresentam quatro dimensões para medir a qualidade da experiência no processo de cocriação de valor, concretamente: a interação social, a interação com os funcionários, o relacionamento com os acompanhantes e o envolvimento do turista com a atividade. Por outro lado, Assiouras et. al. (2019), num painel online composto por turistas gregos, explicam que a cocriação de valor depende de duas dimensões: valor em uso e coprodução. Por sua vez, a coprodução é analisada a partir de três subdimensões, nomeadamente, compartilhamento de conhecimento, equidade e interação. Ainda é de salientar um estudo desenvolvido por Rather et al. (2019), aplicado a um destino turístico famoso na Índia. Para estes investigadores, a cocriação de valor envolve duas dimensões: (i) valor em uso (que é percebido pelo cliente durante as interações de serviço, por exemplo, uso do produto, troca de informações do fornecedor para o cliente) e (ii) coprodução (reflete a inventividade compartilhada, o codesign ou a produção compartilhada). Por fim, num estudo aplicado a uma loja de lembranças para turistas, Sthapit et al., (2018) conceptualizam a cocriação compreendendo dimensões como a participação física e psicológica.

Conforme é possível compreender, encontram-se trabalhos cuja cocriação de valor do cliente é diferentemente conceptualizada, em contextos de estudo similares, por exemplo, no sector hoteleiro os estudos revelam dimensões da cocriação de valor significativamente diferentes (Sarmah, Kamboj \& Rahman, 2017; Shulga et al., 2018; Ahn et al., 2019), assim como a cocriação de valor é analogamente conceptualizada, em contextos de estudo diferentes, por exemplo, entre lojas de venda de lembranças e empresas de turismo de inverno (Prebensen et al., 2016; Sthapit et al., 2018). Isto significa que a comunidade científica é profundamente divergente na concetualização da cocriação de valor e legitima a plasticidade de modelos teóricos entre diferentes contextos de estudo. Com efeito, justifica a criação de um modelo conceptual integrador das diferentes perspetivas, extensível a diferentes domínios da indústria do turismo.

A partir da agregação das várias dimensões identificadas nos estudos realizados sobre a cocriação de valor na perspetiva do S-D Logic nesta indústria, são propostas quatro dimensões fundamentais que permitem compreender genericamente como o cliente é cocriador de valor, conforme se apresenta (Figura 1): 
Figura 1. Principais dimensões da cocriação de valor do turista

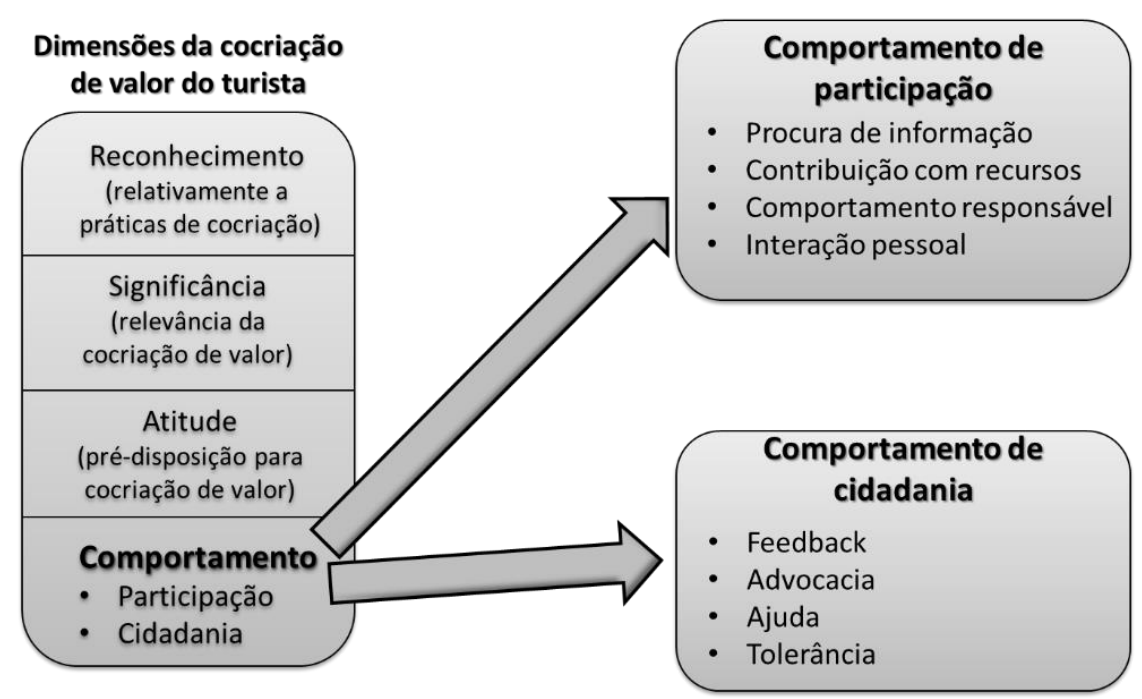

1. Reconhecimento: esta dimensão está ligada à perceção do turista que está a obter reconhecimento intrínseco (não económico, pela partilha de ideias) ou extrínseco (exposto publicamente) por estar implicado com práticas de cocriação de valor (Busser \& Shulga, 2018);

2. Significância, isto é, a crença individual do turista da importância do seu envolvimento e esforço na cocriação de valor. Para o turista é fundamental ter a noção que vale a apena o esforço e o tempo gasto na cocriação de valor (Busser \& Shulga, 2018; Assiouras et al., 2019; Rather et al., 2019);

3. Atitude ou pré-disposição para a cocriação, que deverá compreender três subdimensões: (i) Cognitiva, isto é, como o turista acredita que os seus conhecimentos e habilidades (mestria pessoal) contribuem para o desempenho de um serviço específico (Prebensen \& Xie, 2017); (ii) Afetiva, associada a toda a reacção emocional do turista pela cocriação, manifestando interesse, alegria, felicidade e diversão (Busser \& Shulga, 2018; Prebensen et al., 2016; Sthapit et al., 2018); e (iii) Conativa, ligada à intensão de interação, partilha de conhecimento e resposta favorável do turista relativamente ao prestador de serviços (Ahn et al., 2019; Rather et al., 2019);

4. Comportamento do turista, que poderá ser perspetivado em termos de (i) participação e (ii) cidadania:

No que respeita ao comportamento de (i) participação do turista, é de realçar diferentes formas:

- Procura de informação: o turista recolhe dados para clarificar os requisitos do serviço e reduzir a incerteza ambiental (Ahn et al., 2019; Assiouras et al., 2019);

- Contribuição com recursos: o turista partilha os seus recursos tangíveis e intangíveis ${ }^{1}$ para atingir os resultados desejados (Prebensen et al., 2016; Prebensen \& Xie; 2017; Sarmah, Kamboj \& Rahman, 2017; Busser \& Shulga, 2018; Sthapit et al., 2018; Shulga et al., 2018; Ahn et al., 2019; Assiouras et al. 2019; Rather et al., 2019);

\footnotetext{
${ }^{1}$ A propósito, Hsiao, Lee e Chen (2015) referem que os clientes partilham informação fornecendo aos funcionários da empresa prestadora, informação pessoal, para assegurar que as suas expectativas serão satisfeitas.
} 
- Comportamento responsável: o turista revela que está consciente das suas próprias responsabilidades e tem vontade de cooperar, cumprindo as regras e aceitando as orientações dos funcionários (Sarmah, Kamboj \& Rahman, 2017; Busser \& Shulga, 2018; Shulga et al, 2018; Ahn et al., 2019; Huang \& Choi, 2019);

- Interação pessoal: o turista estabelece interações pessoais com os funcionários, residentes e outros turistas (Prebensen et al., 2016; Sthapit et al., 2018; Shulga et al., 2018; Ahn et al., 2019; Huang \& Choi, 2019; Assiouras et al. 2019; Rather et al., 2019).

Relativamente ao comportamento de (ii) cidadania do turista, é de igual modo de destacar:

- Feedback do turista: o turista fornece activamente sugestões que serão benéficas para a melhoria do serviço (Shulga et al., 2018; Ahn et al., 2019; Assiouras et al., 2019; Rather et al., 2019);

- Advocacia: o turista promove activamente a organização e os seus funcionários junto de pessoas fora da organização, contribuindo para a melhoria da reputação e avaliação de produtos e serviços da organização (Shulga et al., 2018; Ahn et al., 2019; Rather et al., 2019);

- Ajuda: o turista toma a iniciativa de auxiliar outros clientes (Ahn et al., 2019);

- Tolerância: os turistas manifestam maior tolerância em situações em que o serviço prestado pelos funcionários não corresponde às expectativas (Ahn et al., 2019).

O modelo conceptual proposto para além de integrar dimensões e subdimensões recorrentemente utilizadas nos estudos sobre a cocriação de valor do turista na perspetiva do S-D Logic, designadamente a componente comportamental de participação e cidadania (cf. Prebensen et al., 2016; Sthapit et al., 2018; Shulga et al., 2018; Ahn et al., 2019); a intenção comportamental (cf. Ahn et al., 2019; Rather et al., 2019); a resposta afetiva (cf. Busser \& Shulga, 2018; Prebensen et al., 2016; Sthapit et al., 2018) e a significância (cf. Busser \& Shulga, 2018; Assiouras et al., 2019) agrega dimensões e subdimensões menos utilizadas pela comunidade científica, nomeadamente o reconhecimento sobre as práticas de cocriação de valor (cf. Busser \& Shulga, 2018) e a componente cognitiva do turista (cf. Prebensen \& Xie, 2017). Por outro lado, permite observar a cocriação de valor numa perspetiva mais ampla, que transcende a vertente comportamental do cliente (cf. Ahn et al., 2019), reconhecendose a importância da pré-disposição do cliente para a cocriação, assim como, o modo como o cliente reconhece a sua relevância neste processo e o modo como sente que está a ser valorizado por isso (cf. Busser \& Shulga, 2018). Ainda é de salientar que o modelo descrito para além de se harmonizar com diferentes contextos de estudo na indústria do turismo, legitimando a sua ductilidade a partir de outros modelos teóricos igualmente transectoriais testados neste campo (Prebensen et al., 2016; Sthapit et al., 2018), agrega as diferentes conceptualizações da cocriação de valor do turista apresentadas pela comunidade científica, segundo a perspetiva do S-D Logic.

\section{Conclusões e implicações}

O S-D Logic é uma nova abordagem do marketing que assenta na ideia de que a troca consiste no processo das partes usarem o seu conhecimento especializado para benefício de outro. Em termos práticos, significa que as partes (fornecedor-cliente) não trocam produtos e 
serviços por dinheiro, mas trocam um serviço por outro serviço. Por outro lado, esta nova perspetiva realça a premissa de que o valor é determinado pelo cliente através do modo como faz o uso do produto (ou serviço), refutando a noção de "valor utilidade", subjacente em toda a teoria económica desde os seus primórdios. Esta constatação permite realçar que o cliente perceciona o valor do produto, não somente a partir da proposta de valor apresentada pela empresa, como também através do modo como utiliza o produto (ou serviço) no contexto em que está inserido, assim como contribui para a sua produção. Deste modo, o cliente poderá ser encarado como um cocriador e coprodutor de valor.

Dada a abundância de estudos empíricos produzidos pela comunidade científica sobre a cocriação de valor, a escassez de trabalhos que sistematizam os estudos empíricos realizados e, sobretudo, as enormes dissemelhanças identificadas entre as conceções de cocriação de valor na indústria do turismo, urge a necessidade de uma compreensão integradora (holística) do conhecimento empírico e que permita ser facilitadora da sua difusão junto dos principais intervenientes nesta indústria. Assim, tendo por base uma rigorosa revisão dos estudos empíricos realizados na indústria do turismo, recorrendo à perspetiva do S-D Logic e a metodologias quantitativas, neste trabalho apresenta-se um modelo conceptual integrador das diferentes dimensões e subdimensões da cocriação de valor identificadas.

Embora este artigo apresente uma abordagem puramente conceptual, poderá contribuir para uma compreensão mais abrangente das várias dimensões da cocriação de valor do turista, não se cingindo a observar a cocriação de valor numa perspetiva comportamental, associada à participação e coprodução do cliente, vulgarmente conceptualizada na literatura, reconhecendo também a relevância de outras dimensões e do seu papel complementar para a compreensão do conceito de cocriação de valor, designadamente: (i) Atitude ou pré-disposição do turista para a cocriação, em sintonia com uma diversidade de estudos que evidenciam a atitude como um construto multidimensional agregador das três componentes: cognição, afeto e conação (Ajzen, 1993; Hilgar, 1980). Esta dimensão poderá ser particularmente relevante na antevisão de comportamentos do cliente, conforme se evidencia na literatura (cf. Ajzen \& Fishbein, 1977; Ajzen, 1991); (ii) Significância, isto é, a perceção do turista da importância do seu esforço e tempo utilizado na cocriação de valor, pelo valor em uso obtido na experiência de serviço (Rather et al., 2019), poderá ser determinante no comportamento de participação do cliente, como demonstrado por Assiouras et al. (2019); (iii) Reconhecimento pela participação na cocriação de valor. Esta dimensão revela que alguns consumidores são extrinsecamente motivados e exigem compensação material pelas suas ideias e feedback, enquanto outros são motivados intrinsecamente, procurando fama e reconhecimento público. Com efeito, o reconhecimento do turista poderá persuadir o seu comportamento de participação em atividades de cocriação (cf. Kumar et al., 2010).

Mais ainda, é de salientar que este artigo, para além de contribuir para uma explicação mais informada junto de organizações turísticas e planeadores de destinos, sobre a importância do envolvimento do turista na cocriação de valor, poderá contribuir também para uma maior implicação do turista em práticas de cocriação de valor, por uma compreensão mais ampla das dimensões que podem influenciar o seu comportamento (atitude, significância e reconhecimento). Assim, poderá ser estimulada a pré-disposição do turista para a cocriação, a partir de uma comunicação que realce os benefícios da sua participação em atividades de cocriação de valor (por exemplo, salientando-se uma maior 
adaptação e rapidez no serviço prestado e/ou uma diminuição nos custos financeiros do relacionamento). Por outro lado, as organizações poderão proporcionar experiências turísticas cuja proposta de valor seja fortemente determinada pelos recursos tangíveis (ex. esforço físico) e intangíveis (ex. informação) transmitidos pelo cliente, fazendo-o percecionar a importância do tempo e esforço dedicado à organização. Por fim, é ainda de realçar a necessidade das organizações turísticas atenderem a um pacote de incentivos que agregue elementos de índole material (exs. prémios, ofertas ou descontos) e imaterial (ex. reconhecimento público), que vise reconhecer o cliente pelo empenho e tempo dedicado a atividades de cocriação de valor.

Este trabalho almeja também apresentar orientações para futuros trabalhos de investigação, nomeadamente a partir da validação empírica de um questionário que permita medir as dimensões propostas no modelo conceptual de cocriação de valor e possíveis efeitos existentes entre dimensões. Assim, será desejável a operacionalização deste modelo, a partir da realização de estudos empíricos.

\section{Referências}

Ajzen, I. (1991). The theory of planned behavior. Organizational Behavior and Human Decision Processes, 50(2), 179 - 211. doi: 10.1016/0749-5978(91)90020-T

Ajzen, I. (1993). Attitude theory and the attitude-behavior relation. In D. Krebs and P. Schmidt (Eds.), New directions in attitude measurement (pp. 41-57). Berlin: Walter de Gruyter.

Ajzen, I. \& Fishbein, M. (1977). Attitude-behavior relations: A theoretical analysis and review of empirical research. Psychological Bulletin, 84(5), 888-918. doi: 10.1037/0033-2909.84.5.888

Ahn, J., Lee C.-K., Back, K.-J. \& Schmitt, A. (2019). Brand experiential value for integrated resort customers' co-creation behavior. International Journal of Hospitality Management, 81, 104-112. doi: 10.1016/j.ijhm.2019.03.009

Assiouras, I., Skourtis, G., Giannopoulos, A., Buhalis, D., \& Koniordos, M. (2019). Value co-creation and customer citizenship behavior. Annals of Tourism Research, 78, [102742]. doi: 10.1016/j.annals.2019.102742

Beliatskaya, I. (2017). Understanding enhanced tourist experiences through technology: A brief approach to the Vilnius case. Ara: Journal of Tourism Research, 7(1), 17-27.

Berry, L.L. \& Parasuraman, A. (1993). Building a new academic field. The case of service marketing, Journal of Retailing, 69(1), 13 - 60. doi: 10.1016/S0022-4359(05)80003-X

Brodie, R. Glynn, M. S. \& Little, V. (2006). The service brand and the service-dominant logic: Missing fundamental premise or the need for the stronger theory. Marketing Theory, 6(3), 363-379. doi: $10.1177 / 1470593106066797$

Buonincontri, P., Morvillo, A., Okumus, F. \& van Niekerk, M. (2017). Managing the experience co-creation process in tourism destinations: Empirical findings from Naples. Tourism Management, 62(C), 264-277. doi: 10.1016/j.tourman.2017.04.014

Busser, J. A. \& Shulga, L. V. (2018). Co-created value: Multidimensional scale and nomological network. Tourism Management, 65, 69-86. doi: 10.1016/j.tourman.2017.09.014

Campos, A. C., Mendes, J., Oom do Valle, P. \& Scott, N. (2015): Co-creation of tourist experiences: A literature review. Current Issues in Tourism, 21(4), 369-400. doi: 10.1080/13683500.2015.1081158

Cha, M.-K., Yi, Y. \& Bagozzi, R. P. (2016). Effects of customer participation in corporate social responsability (CSR) programs on the CSR-brand fit and brand loyalty. Cornell Hospitality Quarterly, 57(3), 235249. doi: $10.1177 / 1938965515620679$ 
Chandler, J. D. \& Vargo, S. L. (2011). Contextualization and value-in-context: How context frames exchange. Marketing Theory, 11(1), 35-49. doi: 10.1177/1470593110393713

Eiglier, P. \& Langeard, E. (1987). Servuction: Le marketing des services. Paris: Wiley

Chen, Z. (2018). A pilot study of the co-creation experience in traditional Cantonese teahouses in Hong Kong. Journal of Heritage Tourism, 13(6), 506-527. doi: 10.1080/1743873X.2018.1444045

Ge, J. \& Gretzel, U. (2018). A taxonomy of value co-creation on Weibo - A communication perspective. International Journal of Contemporary Hospitality Management, 30(4), 2075-2092. doi: 10.1108/IJCHM-09-2016-0557

Grissemann, U. S. \& Stokburger-Sauer, N. E. (2012). Customer co-creation of travel services: The role of company support and customer satisfaction with the co-creation performance. Tourism Management, 33, 1483-1492. doi: 10.1016/j.tourman.2012.02.002

Grönroos, C. \& Gummesson, E. (1985). The nordic school of services. An introduction. In C. Grönroos and E. Gummesson (Eds.), Service marketing - Nordic School perspectives (pp. 6-11). Stockholm: University of Stockholm.

Grönroos, C. (2006). Adopting a service logic for marketing. Marketing Theory, 6(3), 317-333. doi: $10.1177 / 1470593106066794$

Grönroos, C. (2009). Marketing as promise management: regaining customer management for marketing. Journal of Business \& Industrial Marketing, 24(5/6), 351-359. doi: 10.1108/08858620910966237

Grönroos, C. \& Gummerus, J. (2014). The service revolution and its marketing implications: Service logic Vs service-dominant logic. Managing Service Quality: An International Journal, 24(3), 206-229. doi: 10.1108/MSQ-03-2014-0042

Gummesson, E. (2002). Relationship marketing and the new economy: It's time for deprogramming. Journal of Services Marketing, 16(7), 585-589. doi: 10.1108/08876040210447315

Gummesson, E. \& Mele, C. (2010). Marketing as value co-creation through network interaction and resource integration. Journal of Business Market Management, 4(4), 181-198. doi: 10.1007/s12087-010-0044-2

Hakansson, H. \& Snehota, I. (1995). Developing business relationships in business networks. London: Routledge.

He, W. \& Wang, F.-W. (2016). A process-based framework of using social media support innovation process. Information Technology and Management, 17, 263-277. doi: 10.1007/s10799-0150236-2

Horbel, C. (2013). Service-dominant logic and tourism management. Enriching each other. Die Betriebswirtschaft, 73(2), 131-142.

Hsiao, C. Lee, Y.-H. \& Chen, W.-J. (2015). The effect of servant leadership on customer value co-creation: A cross-level analysis of key mediating roles. Tourism Management, 49, 45-57. doi: 10.1016/j.tourman.2015.02.012

Huang, S. \& Choi, H.-S. C. (2019). Developing and validating a multidimensional tourist engagement scale (TES). The Service Industries Journal, 39(7-8), 469-497. doi: 10.1080/02642069.2019.1576641

Hunt, S. (2000). A general theory of competition: Resources, competences, productivity, economic growth. Thousand Oaks, CA: Sage Publications.

Kumar, V., Aksoy, L., Donkers, B., Venkatesan, R., Wiesel, T. \& Tillmanns, S. (2010). Undervalued or overvalued customers: Capturing total customer engagement value. Journal of Service Research, 13(3), 297-310. doi: 10.1177/1094670510375602

Malone, S., McKechnie, S. \& Tynan, C. (2018). Tourists' emotions as a resource for customer value creation, co-creation and destruction: A customer-grounded understanding. Journal of Travel Research, 57(7), 843-855. doi: 10.1177/0047287517720118 
Mele, C., Pels, J. \& Storbacka, K. (2015). A holistic market conceptualization. Journal of the Academy of Marketing Science, 43(1), 100-114. doi: 10.1007/s11747-014-0383-8

Merz, M., He, Y. \& Vargo, S. L. (2009). The evolving brand logic: A service-dominant logic perspective. Journal of the Academy of Marketing Science, 37(3), 328-344. doi: 10.1007/s11747-009-0143-3

Park, S.-Y. \& Vargo, S.L. (2012). The service-dominant logic approach to tourism marketing strategy. In R. H. Tsiotsou and R. E. Goldsmith (Eds.), Strategic marketing in tourism services (pp. 231-246). UK: Emerald Group Publishing Limited.

Prahalad, C.K. \& Hamel, G. (1990). The core competence of the corporation. Harvard Business Review, 68, 79-91.

Prebensen, N. K. \& Dahl, J. V. T. (2013). Value co-creation significance of tourist resources. Annals of Tourism Research, 42, 240-261. doi: 10.1016/j.annals.2013.01.012

Prebensen, N. K., Woo, E. \& Uysal, M. S. (2013). Experience value: Antecedents and consequences. Current Issues in Tourism, 17(10), 910-928. doi: 10.1080/13683500.2013.770451

Prebensen, N. K., Kim, H. L. \& Uysal, M. (2016). Cocreation as Moderator between the Experience Value and Satisfaction Relationship. Journal of Travel Research, 55(7), 934-945. doi: 10.1177/0047287515583359

Prebensen, N. \& Xie, J. (2017). Efficacy of co-creation and mastering on perceived value and satisfaction in tourists' consumption. Tourism Management, 60, 166-176. doi: 10.1016/j.tourman.2016.12.001

Rather, R. A., Hollebeek, L. D. \& Islam, J.U. (2019). Tourism-based customer engagement: The construct, antecedents and consequences. The Service Industry Journal, 39(7-8), 519-540. doi: 10.1080/02642069.2019.1570154

Rihova, I., Buhalis, D., Moital, M. \& Gouthro, M. B. (2015). Conceptualising customer-to-customer value co-creation in tourism. International Journal of Tourism Research, 17, 356-363. doi: 10.1002/jtr.1993

Rihova, I., Buhalis, D., Gouthro, M. B., \& Moital, M. (2018). Customer-to-customer co-creation practices in tourism: Lessons from customer-dominant logic. Tourism Management, 67, 362 - 375. doi: 10.1016/j.tourman.2018.02.010

Sarmah, B., Kamboj, S. \& Rahman, Z. (2017). Co-creation in hotel service innovation using smart phone apps: an empirical study. International Journal of Contemporary Hospitality Management, 29 (10), 2647 - 2667. doi: 10.1108/IJCHM-12-2015-0681

Shamim, A. \& Ghazali, Z. (2014). A conceptual model for developing customer value co-creation behaviour in retailing. Global Business and Management Research: An International Journal, 6(3), 185-196.

Shaw, G. Bailey, A. \& Williams, A. (2011). Aspects of service-dominant logic and its implications for tourism management: Examples from the hotel industry. Tourism Management, 32, 207-214. doi: 10.1016/j.tourman.2010.05.020

Shostack, G. L. (1977). Breaking free from product marketing. Journal of Marketing, 41(2), 73-80. doi: $10.2307 / 1250637$

Shulga, L. V., Busser, J.A. \& Kim, H. L. (2018). Generational profiles in value co-creation interactions. Journal of Hospitality Marketing \& Management, 27(2), 196-217. doi: 10.1080/19368623.2017.1340218

Skålén, P. Gummerus, J. Koskull, C. \& Magnusson, P. (2015). Exploring value propositions and service innovation: A service-dominant logic study. Journal of the Academy of Marketing Science, 43(2), 137-158. doi: 10.1007/s11747-013-0365-2

Smith, A. [1776] (2007). An inquiry into the nature and causes of the wealth of nations. Ed. S.M. Soares. MetaLibri Digital Library. 
Sthapit, E., Coudounaris, D. N. \& Björk, P. (2018). The memorable souvenir-shopping experience: Antecedents and outcomes. Leisure Studies, 37(5), 628-643. doi: 10.1080/02614367.2018.1519031

Tuan, L. T., Rajendran, D., Rowley, C. \& Khai, D. C. (2019). Customer value co-creation in the business-tobusiness tourism context: The roles of corporate social responsability and customer empowering behaviors. Journal of Hospitality and Tourism Management, 39, 137-149. doi: 10.1016/j.jhtm.2019.04.002

Vargo, S. L. \& Akaka, M. A. (2009). Service-dominant logic as a foundation for service science: Clarifications. Service Science, 1(1), 32-41. doi: 10.1287/serv.1.1.32

Vargo, S. L., Koskela-Huotari, K., Baron, S., Edvardsson, B., Reynoso, J. \& Colurcio, M. (2017). A system perspective on markets. Toward a research agenda. Journal of Business Research, 79, 260-268. doi: 10.1016/j.jbusres.2017.03.011

Vargo, S. L. \& Lusch, R. (2004). Evolving to a new dominant logic for marketing. Journal of Marketing, 68, 1-17. doi: 10.1509/jmkg.68.1.1.24036

Vargo, S. L. \& Lusch, R. (2006). Service-dominant logic: What it is, what is not, what it might be. In R.F. Lush and S. L. Vargo (Eds.), The service-dominant logic of marketing: Dialog, debate, and directions (pp. 43 - 56). New York: M. E. Shape.

Vargo, S. L. \& Lusch, R. (2008). Service-dominant logic: Continuing the evolution. Journal of the Academy of Marketing Science, 36, 1-10. doi: 10.1007/s11747-007-0069-6

Vargo, S. L. \& Lusch, R. (2013). Service-dominant logic: Prologue and prospects. Die Betriebswirtschaft, 73(2), 91-93.

Vargo, S. L. Lusch, R., Akaka, M. A. \& He, Y. (2010). Service dominant-logic: A review and assessement. Review of Marketing Research, 6, 125-167. doi: 10.1108/S1548-6435(2009)0000006010

Vargo, S. L., Lusch, R. Horbel, C \& Wieland, H. (2011). Alternative Logics for Service(S): From Hybrid Systems to Service Ecosystems. In D. Spath and W. Ganz (eds.), Taking the Pulse of Economic Development - Service Trends (pp. 123 - 135). Munich: Hanzer Verlag.

Wieland, H., Hartmann, N. N. \& Vargo, S. L. (2017). Business models as service strategy. Journal of the Academy of Marketing Science, 45, 925-943. doi: 10.1007/s11747-017-0531-z

Woratschek, H. \& Horbel, C. (2005). Are variety seekers bad customers? An analysis of the role of recommendations in the service profit chain. Journal of Relationship Marketing, 4(3-4), 43-57. doi: 10.1300/J366v04n03_04

$\underline{\mathrm{Xu}}, \mathrm{H}$. Liu, Y. \& Lyu, X. (2018). Customer value co-creation and new service evaluation: The moderating role of outcome quality. International Journal of Contemporary Hospitality Management, 30(4), 2020-2036. doi: 10.1108/IJCHM-08-2016-0467

Yi, Y. \& Gong, T. (2013). Customer value co-creation behavior: Scale development and validation. Journal of Business Research, 66(9), 1279-1284. doi: 10.1016/j.jbusres.2012.02.026

PEDRO MIGUEL CARVALHO é doutorado em gestão de empresas na especialidade do marketing, pela Universidade da Extremadura (Espanha). É professor adjunto no Instituto Politécnico de Viana do Castelo, onde tem lecionado nas áreas do marketing e gestão a cursos de licenciatura e mestrado. Tem também desenvolvido investigação em marketing turístico no Centro de Investigação, Desenvolvimento e Inovação em Turismo (CITUR). Endereço institucional: Instituto Politécnico de Viana do Castelo, Escola Superior de Tecnologia e Gestão, Avenida do Atlântico, n. ${ }^{\circ}$ 644, 4900-348 Viana do Castelo, Portugal.

Submetido em 12 de fevereiro de 2020

Aceite em 7 de maio de 2020 